\title{
Two Three-dimensional Metal-organic Frameworks Containing \\ One-dimensional Hydroxyl/Carboxylate Mixed bridged Metal Chains: Syntheses, Crystal Structures and Magnetic Properties
}

\author{
Xiao-Ju Li ${ }^{\dagger}$ Xin-Yi Wang, ${ }^{\star}$ Song Gao $,{ }^{*},+$ and Rong Cao $*, \dagger$
}

State Key Laboratory of Structural Chemistry, Fujian Institute of Research on the Structure of Matter, The Chinese Academy of Sciences, Fuzhou, Fujian 350002, P. R. China; State Key Laboratory of Rare Earth Materials Chemistry and Applications, College of Chemistry and Molecular Engineering, Peking University, Beijing 100871, P. R. China

* Authors to whom correspondence should be addressed. E-mail: gaosong@pku.edu.cn (S. G.); rcao@,fjirsm.ac.cn (R. C.). Fax: +86-591-83714946.

\section{Supporting Information}

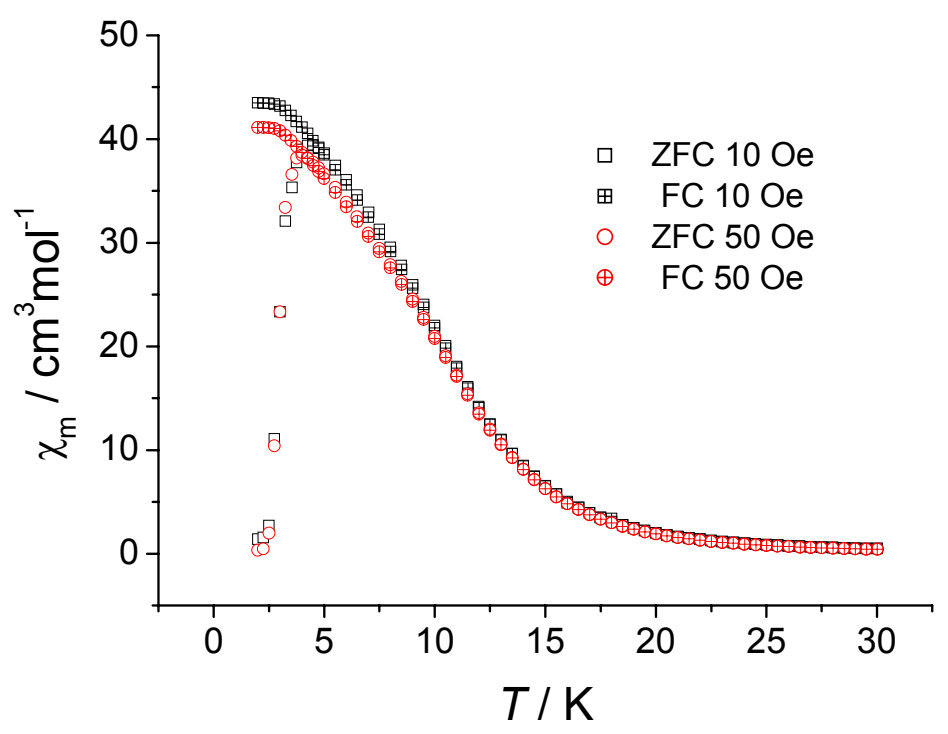

Figure S1. Temperature dependence of zero-field-cooling (ZFC) and field-cooling (FC) $\chi_{\mathrm{m}}$ of 1 . 


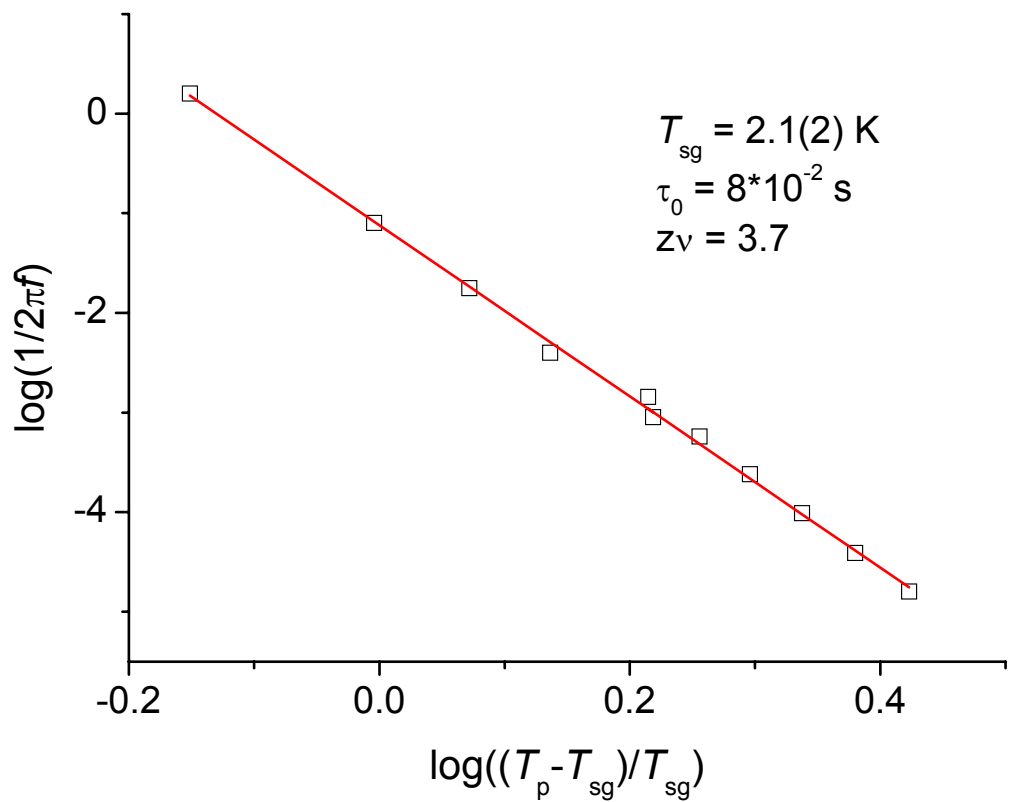

(a)

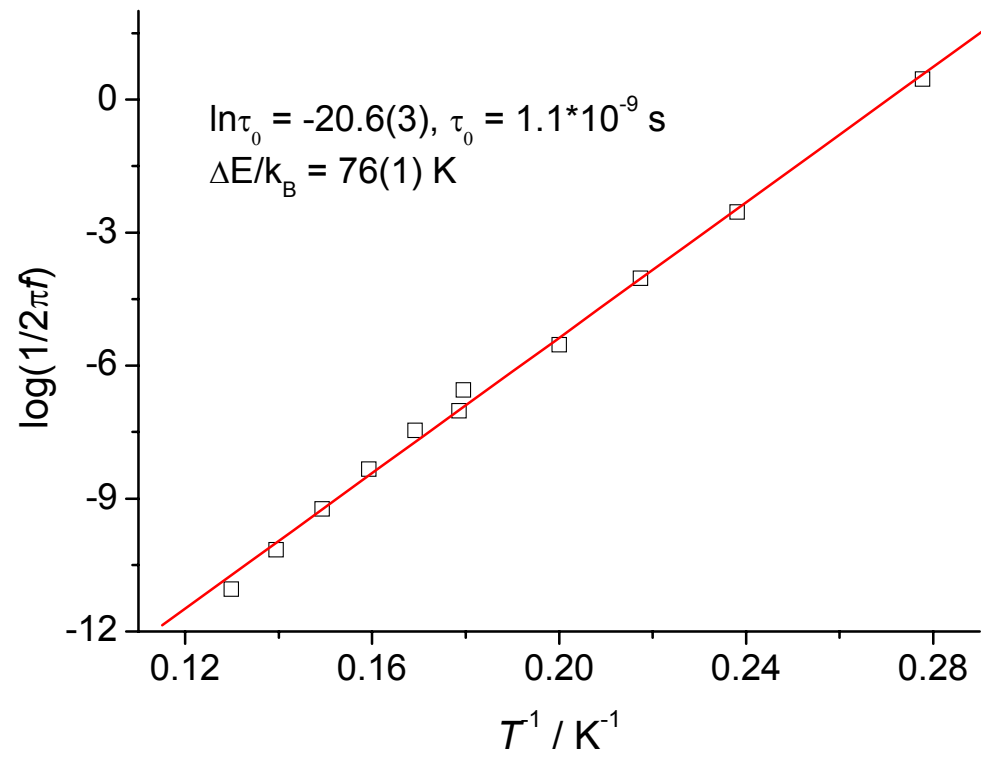

(b)

Figure S2. (a) Frequency dependence of ac $\chi$ " for 1 was fitted by the conventional critical scaling law of the spin dynamics as described by $\tau=\tau_{0}\left(\left(T_{\mathrm{P}}-T_{\mathrm{f}}\right) / T_{\mathrm{f}}\right)^{-z v}$; (b) Frequency dependence of ac $\chi$ ” for 1 was fitted by Arrhenius law $\tau=\tau_{0} \exp (\Delta E / \mathrm{kB} T)$. 


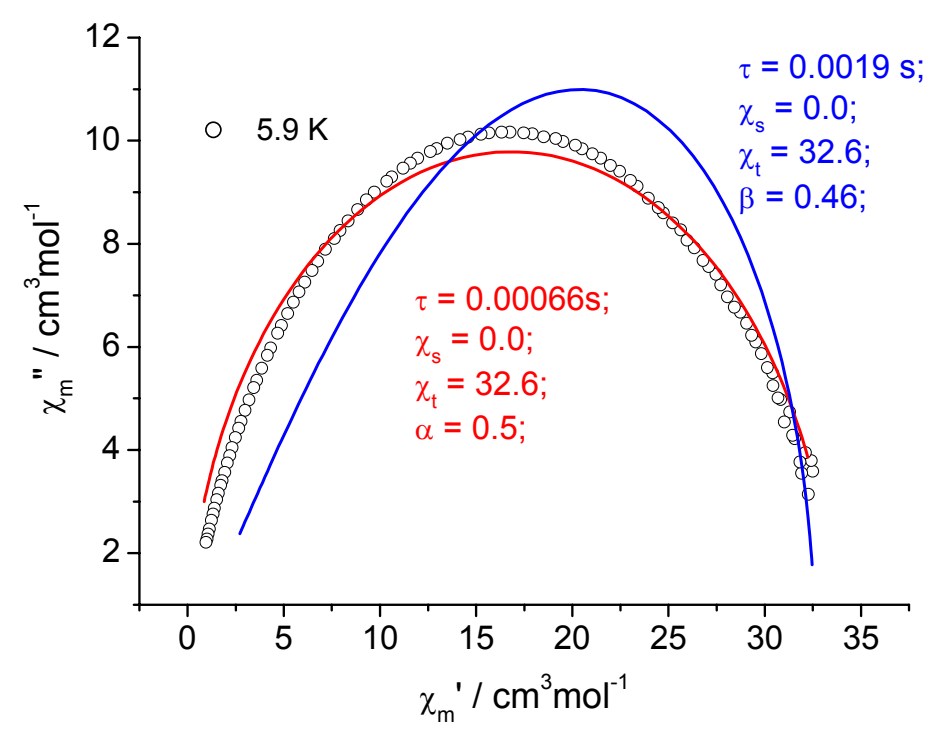

Figure S3. Cole-Cole diagram of $\mathbf{1}$ at $5.9 \mathrm{~K}$ with zero dc field. The red solid line is least-squares fitting of the data to a distribution of single relaxation processes with a generalized Debye model, giving parameter values: $\chi_{\mathrm{s}}=0.0 \mathrm{~cm}^{3} \mathrm{~mol}^{-1}, \chi_{\mathrm{t}}=32.6$ $\mathrm{cm}^{3} \mathrm{~mol}^{-1}, \tau=0.00066 \mathrm{~s}, \alpha=0.5$. (Refs. Cole, K. S.; Cole, R. H. J. Chem. Phys. 1941, 9, 341. Aubin, S. M.; Sun, Z.; Pardi, L.; Krzysteck, J.; Folting, K.; Brunel, L. J.; Rheingold, A. L.; Christou G.; Hendrickson, D. N. Inorg. Chem. 1999, 38, 5329.) The blue line is the least-squares fitting of the data to a Cole-Davidson equation, giving parameter values: $\chi_{\mathrm{s}}=0.0 \mathrm{~cm}^{3} \mathrm{~mol}^{-1}, \chi_{\mathrm{t}}=32.6 \mathrm{~cm}^{3} \mathrm{~mol}^{-1}, \tau=0.0019 \mathrm{~s}, \beta=0.46$. (Ref. Davision, D. W.; Cole, R. H. J. Chem. Phys. 1951, 19, 1484.) 